\title{
COMPUTER ALGEBRA IN NONLINEAR ANALYSES OF THE STRAIGHTLINE STABILITY OF COMBINATION VEHICLES
}

\author{
M. W. SAYERS $\dagger$ and A. STRIBERSKY $\ddagger$ \\ †Transportation Research Institute, The University of Michigan, 2901 Baxter Road, Ann Arbor, \\ MI 48109, U.S.A. \\ $\ddagger$ Research and Development, SGP Verkehrstechnik Gesellschaft m.b.H., Brehmstraße 16, \\ A-1110 Vienna, Austria
}

\begin{abstract}
Recent developments in the theories of the dynamics of nonlinear systems and bifurcations provide a means for studying nonlinear oscillations and stability problems. The analysis methods require that equations of motion for the system of interest be derived in a certain form. Such derivation can involve substantial algebraic manipulation. However, by using recently developed computer algebra software, the entire derivation can be performed automatically by a computer. The level of automation associated with the analysis makes it practical for general use by engineers. An application from vehicle system dynamics is given as an example.
\end{abstract}

\section{INTRODUCTION}

Many engineering problems require both symbolic and numerical analysis to gain insight into the behavior of some system of interest. That is, a specific system is analyzed symbolically, and as a result of the analysis, an algorithm is selected to perform an appropriate numerical procedure. Automated numerical analyses are routinely applied by computer to obtain quantitative answers. In contrast, symbolic analysis are commonly still performed with pencil, paper, and the knowledge of algebra and calculus. With these tools, a symbolic analysis involving more than a few hundred steps becomes a large and tedious undertaking that requires considerable motivation and time on the part of a human analyst.

This paper describes a type of study in which substantial symbolic analysis is necessary. Specifically, we will describe how new computer methods are used to perform the extensive symbolic manipulations needed to apply bifurcation theory for investigating the stability of tractor-semitrailers and combination vehicles involving multiple trailers. Because the derivation of the required equations can involve thousands of steps, the methodology requires that the symbolic manipulations be performed automatically by computer.

The study is motivated by the observation that ground vehicles can experience a flutter instability at high forward speeds, whether they are heavy-duty truck combinations [1] or railway vehicles [2]. Several new developments in nonlinear dynamics theory [3-5] and in computer algebra [6] provide tools that can now be applied by the vehicle engineer to gain more insight into this problem.

When certain combination vehicles are driven in a straightline motion at high forward speeds, self- excited yaw oscillations can be initiated. The steadystate straightline motion can lose its stability due to a bifurcation of a limit cycle from the original equilibrium position. The stability boundary can be readily calculated with linear methods, but the analysis of the behavior at the limit requires nonlinear methods. A critical question is whether the bifurcating periodic motion is stable or unstable. For the case where the limit cycle branch shows a limit point, a global analysis becomes necessary. The application of the methods of bifurcation theory allows a nonlinear stability analysis to be performed in a systematic manner. Detailed numerical and experimental results are presented in the following papers: $[1,7,8]$. Throughout this paper we concentrate on the computational aspects.

\section{MECHANICAL MODEL}

For the truck combinations of interest, a heavyduty tractor is towing one or more trailers. (See for example the mechanical model of a twin-trailer truck in Fig. 1.) To study the yaw stability of such articulated vehicles on a horizontal flat road, a simplified rigid body model can be used. This model neglects the roll, pitch and bounce motions of the sprung masses. We always consider symmetric vehicle combinations. The tractor is assumed to move forward with a constant forward speed, $V$. The forward speed is the main parameter in the system. Additional parameters are the loading conditions of the trailers, which are expressed by the distances $d_{1}$ and $d_{2}$, respectively. The investigations are done for open-loop systems with a fixed steering wheel $(\delta \equiv 0)$.

To calculate the lateral tire forces, $S_{i}$, we use the static tire vertical loads, $Q_{i}$, and apply an adhesion/sliding approach proposed by Schallamach 


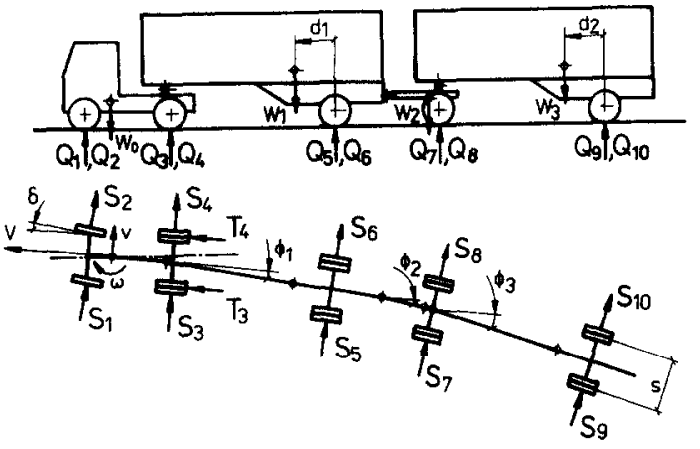

Fig. 1. Mechanical model of a combination vehicle with five degrees of freedom $\left(v, \omega, \phi_{1}, \phi_{2}, \phi_{3}\right)$.

and Turner [9] that accounts for tire slip angle, $\alpha$, and vertical tire load, $Q$, at each wheel

$$
\begin{aligned}
S=\frac{\mu Q}{\pi}\left[\frac{2 K \tan \alpha}{1+K^{2}(\tan \alpha)^{2}}\right. & \\
& \left.+\arcsin \frac{2 K \tan \alpha}{1+K^{2}(\tan \alpha)^{2}}\right]
\end{aligned}
$$

where $K=\left(\pi C_{\alpha} / 4 \mu Q\right)$.

The cornering stiffness, $C_{x}$, and the friction level, $\mu$, are tire-parameters that are set to match experimental data. The dependence of the cornering stiffness on the vertical tire load is expressed by an empirical formula. The friction level is assumed to decrease linearly as the vertical force increases. The slip angles are obtained from the kinematics of the system. For the trailing units they are highly nonlinear expressions, which involve many of the state variables.

\section{NONLINEAR EQUATIONS OF MOTION FOR MECHANICAL SYSTEMS}

To investigate the stability behavior for the vehicle systems of interest, we need equations of motion in the form:

$$
\begin{aligned}
\dot{\mathbf{y}}=\mathbf{f}(\mathbf{y}, \lambda)=\mathbf{A}(\lambda)+\mathbf{B}(\lambda) \mathbf{a}^{3}(\mathbf{y}) & \\
& +\mathbf{C}(\lambda) \mathbf{a}^{5}(\mathbf{y})+O\left(|\mathbf{y}|^{\gamma}\right),
\end{aligned}
$$

where $y$ is the vector of state variables defined locally with respect to an equilibrium position, and $\lambda$ is the vector of parameters. The vectors $\mathbf{a}^{i}(y)$ contain only polynomials of order $i$ in the state variables, e.g. $\left[\mathbf{a}^{3}(y)\right]^{T}=(y 1 * y 1 * y 1, y 1 * y 1 * y 2, y 1 * y 1 *$ $y 3, \ldots)$. Due to the symmetry of the vehicle combinations, only third and fifth order terms appear as nonlinearities. Equation (2) can be derived by power series expansions in terms of the local state variables in respect to the steady-state straightline motion. To perform a local stability analysis, the expansion must include terms up to the third power. For a global stability investigation, terms up to the fifth power are needed.
For very simple vehicle models, eqn (2) can be derived manually. However, a considerable amount of algebraic manipulation is needed even for models with only a few degrees of freedom. Alternatively, a symbolic multibody program can be used to aid in the analysis.

There is a large body of literature covering systematic analysis methods for mechanical systems composed of rigid bodies [10-15]. In most of these methods, called 'multibody formalisms', the equations of motion are essentially defined for once and for all in generic form. The analysis for a specific multibody system consists of plugging in dimensions and inertia parameters in the appropriate spots in the equations. Most of the formalisms that have been developed could conceivably be implemented either numerically or symbolically.

For the analyses reported in this paper, a software package called AUTOSIM was used to automatically generate symbolic equations in the exact form of eqn (2). AUTOSIM was developed to form equations of motion for multibody systems and generate simulation codes that numerically solve the equations using numerical simulation algorithms [6]. It also includes some techniques that are well suited for obtaining the power series expansion of eqn (2).

To start a multibody analysis with AUTOSIM, we first have to describe the mechanical system in AUTOSIM notation. For example, Fig. 2 shows a sketch that describes a model of a tractor-semitrailer vehicle. The figure shows two rigid bodies, $\mathbf{A}$ and $\mathbf{B}$, that move in a plane parallel to the road. Each body has its own coordinate system, whose origin is a point named by appending ' 0 ' to the symbol of the body. Also, each body has a point for its center of mass. For body $A$, the origin and center of mass are the same point. For body B, the origin is the hitch location, which differs from the center of mass. Axes of the coordinate system of $A$ are in directions defined by unit-vectors $\mathbf{a}_{1}, \mathbf{a}_{2}$, and $\mathbf{a}_{3}$. The third of these, $\mathbf{a}_{3}$, is defined as $\mathbf{a}_{1} \times \mathbf{a}_{2}$, and is parallel to the vertical direction fixed in the inertial reference, $\mathbf{n}_{3}$. A similar convention is used for body B.

The bodies are acted on by six forces due to tire-road interactions: $S_{1}, \ldots, S_{6}$. The forces are applied at points labelled $\mathrm{Pl}, \ldots, \mathrm{P} 6$, as shown in the

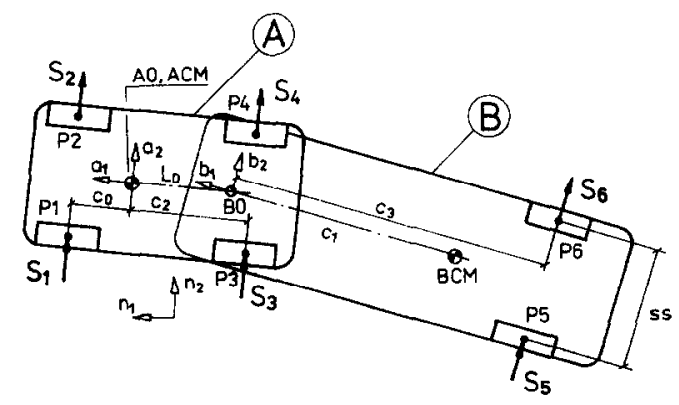

Fig. 2. Rigid-body representation of a tractor-semitrailer. 
figure. The lateral tire forces are defined by the mentioned adhesion/sliding model [eqn (1)].

The AUTOSIM analysis then involves six steps: (1) describe the system, (2) constraint analysis, (3) kinematics analysis, (4) dynamics analysis, (5) uncouple the equations of motion, and (6) write the equations of motion.

The six steps performed by AUTOSIM to obtain the equations of motion have been described in detail elsewhere [6] and are only viewed briefly below in the context of the tractor-semitrailer vehicle model. Note that the multibody analysis is performed symbolically only once, using symbols for vehicle and tire parameters. Thus, the resulting equations can be used for any set of parameter values.

\section{Step 1. Describe the system}

In this step, an input file is prepared to communicate the model to AUTOSIM using commands such as 'add-body', 'add-point', 'add-constraint', and 'add-line-force'. Also, global variables are set to enable and disable options involving the symbolic manipulation. The entire description for the tractor-semitrailer model is listed in the appendix. Bodies, points, and directions appearing in the appendix are shown in Fig. 2.

An important feature of AUTOSIM is that it is easily extended. Just as AUTOSIM was written in Common Lisp [16] to extend Lisp, additional software can be written in Lisp to extend AUTOSIM. A case in point is a new macro called 'add-tire' that was added for this analysis to simplify the vehicle description. Given a point $P$ as an argument, the macro develops a symbolic expression for $\tan \alpha$, where $\alpha$ is the angle between the forward direction of the body containing $P$ and the velocity of $P$. The expression for $\tan \alpha$ is expanded and terms equal or above the specified threshold are dropped. Equation (1) is then applied to develop an expression for the magnitude of the lateral tire force. The force is applied to the body containing $P$ via the AUTOSIM function 'add-lineforce*'.

As each body is entered (via the 'add-body' command), generalized coordinates and generalized speeds are introduced automatically to account for degrees of freedom of the joint connecting the new body to its parent. (The parent is either another body or the inertial reference.) A set of three unit-vectors is introduced to define directions of the axes of a coordinate system fixed in the new body. A direction cosine matrix is formed to relate the unit-vector fixed in the new body to the unit-vectors of the parent.

The example system has four degrees of freedom after the two 'add-body' commands are processed. Each degree of freedom is characterized by a generalized speed. The four speeds are: forward and side velocity of the tractor mass center, yaw rate of the tractor, and the rate of the articulation angle between tractor and trailer.

\section{Step 2. Constraint analysis}

In addition to constraints defined by the joints connecting bodies to their parents, the multibody system might be subject to additional constraints. In the case of the example tractor-semitrailer system, there is one such constraint: the forward speed of the tractor is constant. This information was provided with the 'add-constraint' command, that states that the forward speed minus a constant is equal to zero. Each constraint equation is used to symbolically eliminate one state variable, selected automatically based on criteria for minimizing the likelihood of a singularity. Thus, the example system has three degrees of freedom after the constraint is added. (The forward speed is eliminated as a state variable and will be the main parameter in the bifurcation analysis.)

Most of the constraint analysis is actually performed when the 'add-constraint' command is processed. A few remaining steps are performed when the 'dynamics' command is processed, to define coefficients used in the dynamics analysis.

\section{Step 3. Kinematics analysis}

A set of differential equations is formed to define derivatives of coordinates in terms of speeds

$$
\dot{\mathbf{q}}=\mathbf{g}(\mathbf{q}, \mathbf{u}) \text {, }
$$

where $q$ is a one-dimensional array of generalized coordinates, is an array of their derivatives, and $n$ is an array of generalized speeds. These equations are based solely on kinematical considerations, and are called kinematical equations. In the example, four equations are formed to define the derivatives of the generalized coordinates in terms of the three independent generalized speeds.

\section{Step 4. Dynamics analysis}

A set of implicit differential equations is obtained using a rule-based method derived from Kane's equations [17]. These equations are represented in matrix form as follows:

$$
\mathbf{M}(\mathbf{q}) \dot{\mathbf{u}}=\mathbf{f}^{\prime \prime}(\mathbf{q}, \mathbf{u}),
$$

where $\mathbf{M}$ is the mass matrix and $\mathbf{f}^{\prime \prime}$ is a force array. They are called the dynamical equations, and satisfy the Newton and Euler equations for a dynamical mechanical system. The dynamical equations include references to the articulation angle, $q_{4}$, but not any of the other three generalized coordinates. For this reason, the three kinematical equations for the other coordinates are not needed in the stability analysis.

\section{Step 5. Uncouple the equations}

The dynamical equations are linear with respect to $u$ and can therefore be uncoupled symbolically using linear algebra. In AUTOSIM, Crout's algorithm is 
used to perform lower-upper decomposition (LUD) of the mass matrix. Forward-backward substitution is then used to obtain the desired form of the equations

$$
\grave{\mathbf{u}}=\mathbf{M}(\mathbf{q})^{-1} \mathbf{f}^{\prime \prime}(\mathbf{q}, \mathbf{u})=\mathbf{f}^{\prime}(\mathbf{q}, \mathbf{u}) .
$$

In normal use of AUTOSIM, the array $f^{\prime}$ is not obtained. Instead, a recursive set of equations is derived in which expressions for derivatives of generalized speeds can include other derivatives that have already been defined. The recursion results in simpler equations that lead to better computational efficiency in a simulation code. However, for nonlinear stability analyses, a nonrecursive form is needed. It is obtained by expanding any derivatives appearing on the righthand side of the equations, to define elements in $r$ that are functions only of $q$ and $u$. (That is, $f$ is not a function of $\mathbf{u})$.

\section{Step 6. Print equations}

The final step is to print the equations. Depending on which simplification options were in use when the equations were derived, some terms may have been derived which are not required to compute the derivates in eqn (3) or (5). These equations are removed. In normal use, the equations are printed in the middle of a self-contained computer program that simulates the system of interest by numerically integrating the equations of motion. However, it is also possible to print just the equations and use them for other purposes, as was done for the analyses described in this paper.

The kinematical and dynamical equations are combined into the form of eqn (2) by defining

$$
\mathbf{y}^{T}=\left[\mathbf{q}^{T} \mathbf{u}^{T}\right], \quad \mathbf{f}^{T}=\left[\mathbf{g}^{T} \mathbf{f}^{T}\right] .
$$

\section{THE METHOD OF THE NONLINEAR STABILITY ANALYSIS}

The straightline stability of combination vehicles is examined using the methods of bifurcation theory, as it is described by Stribersky et al. [8]. These methods require that the equations of motion for the vehicle system be expressed as sums of linear terms and nonlinear terms in the form of eqn (2), e.g. for a twin-trailer truck, the vector $\mathbf{y}$ of state variables is defined as: $\mathbf{y}^{T}=\left(\phi_{1}, \phi_{2}, \phi_{3}, v, \omega, \phi_{1}, \phi_{2}, \phi_{3}\right)$. The parameter vector is defined as $\lambda^{T}=\left(V, d_{1}, d_{2}\right)$. The straightline motion $y_{0} \equiv 0$, which gives $\mathbf{0}=\mathbf{f}(0, \lambda)$, is an equilibrium position of the system for all values of $\lambda$.

The stability of the equilibrium $y_{0}$ is guaranteed by a theorem of Liapunov, if all eigenvalues of the matrix $\mathbf{A}(\lambda)$ of eqn (2) have negative real parts. Starting with a stable straightline motion at a low driving speed, $V$, we increase the driving speed at fixed loading conditions quasi-statically and calculate the eigenvalues of the matrix $\mathbf{A}(\lambda)$. The stability boundary is reached if one or more eigenvalues crosses the imaginary axis. One purely imaginary pair of eigenvalues at the critical speed indicates a futter instability caused by a Hopf bifurcation.

A linear transformation of variables $y \rightarrow z$ of the form $y=T z$ transforms the linear part of eqn (2) into diagonal form. The matrix $T$ is given by the eigenvectors of $\mathrm{A}(\lambda)$. At the critical equilibrium point $\left(V_{c}\right)$ we can reduce the originally high-dimensional system to a low dimensional bifurcation system by using center manifold theory [4]. For the cases considered, the bifurcation system is two-dimensional, because at the critical speed, $V_{c}$, one pair of complex eigenvalues crosses the imaginery axis. To transform the bifurcation equation to its simplest form, we apply the averaging principle up to the fifth order terms $[18,19]$. For generic cases of loading conditions, we end up with the normal form of a Hopf bifurcation. Higher than third order terms can then be neglected. In polar coordinates, the equation for the averaged amplitude becomes:

$$
r=v_{1} r+K_{3} r^{3}+O\left(|r|^{5}\right)
$$

Equation (7) has already been unfolded with the mathematical unfolding parameter

$$
v_{1}=\left(\frac{\partial \sigma_{1}}{\partial \lambda_{1}}\right)_{\lambda_{1}=0} i_{1}
$$

where $\sigma_{1}$ is the real part of the critical eigenvalues, and $\lambda_{1}=V-V_{r}$.

For a twin-trailer truck, we can adjust the loading condition of the second trailer to a point where a subcritical Hopf bifurcation becomes supercritical. This enables us to perform a global stability analysis. Such analysis includes the fifth order terms. The unfolding of the bifurcation equation for the nongeneric case $K_{3}=0$ leads to the normal form of a generalized Hopf bifurcation

$$
\dot{r}=v_{1} r+v_{2} r^{3}+K_{5} r^{5}+O\left(|r|^{7}\right),
$$

where

$$
v_{1}=\left(\frac{\partial \sigma_{1}}{\partial \epsilon_{1}}\right)_{\epsilon_{1}=0, \epsilon_{2}=0} \epsilon_{1}, \quad v_{2}=\left(\frac{\partial K_{3}}{\partial \epsilon_{2}}\right)_{k_{1}=0, \epsilon_{2}=0} \epsilon_{2} .
$$

Figure 6 shows the linear relation of the unfolding parameters (directions $\epsilon_{1}, \epsilon_{2}$ ) to the physical parameters $\left(\lambda_{1}=V-V_{c}, \lambda_{2}=d_{2}-d_{2,0}\right)$.

\section{SYMBOLIC COMPUTATION METHODS}

A number of symbol manipulation techniques are employed in AUTOSIM to produce equations of motion that are very efficient for computing derivatives of state variables. Four of these techniques are: (1) products of expressions are left in factored form, 


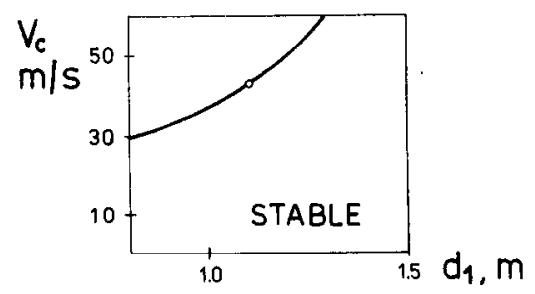

Fig. 3. Stability boundary in the parameter space.

(2) intermediate variables are introduced for expressions that appear more than once in the equations, (3) constant expressions are identified and 'pre-computed', and (4) knowledge that some variables and constants are 'small' is used to drop higher-order terms and replace functions with truncated power series. The first two techniques are not used when coefficients in front of the various powers of the state variables are needed. To obtain these coefficients, products of expressions are always expanded, and intermediate variables are not introduced for expressions unless they are constant.

The symbolic equations needed for the bifurcation analysis are rather complicated. For example, the three dynamical equations for the simple three DOF tractor-semitrailer model involve over 1000 combinations of constants when the power series are expanded to include third order terms. To given an idea of where the complexity arises, consider the expression developed for the side force for one of the rear tires of the tractor-semitrailer vehicle

$$
\begin{aligned}
S_{6}= & -\left(p_{239} q_{4}^{3}+p_{173} q_{4}+p_{177} q_{4} u_{2}+p_{180} u_{2}^{2}\right. \\
& +p_{299} q_{4} u_{2}^{2}+p_{298} u_{2}^{3}+p_{178} u_{2}+p_{236} u_{2} q_{4}^{2} \\
& -p_{233} q_{4} u_{2} u_{1}-p_{189} u_{1}-p_{297} u_{1} u_{2}^{2} \\
& -p_{230} u_{1}^{3}-p_{232} u_{2} u_{1}^{2}-p_{190} u_{2} u_{1} \\
& -p_{235} u_{1} q_{4}^{2}+p_{234} q_{4} u_{1}^{2}+p_{294} u_{3} u_{2}^{2} \\
& -p_{229} u_{3} u_{1}^{2}+p_{212} u_{3}+p_{177} q_{4} u_{3} \\
& +p_{213} u_{3}^{2}-p_{225} q_{4} u_{1} u_{3}-p_{296} u_{1} u_{3}^{2} \\
& +p_{224} u_{3} q_{4}^{2}+p_{293} q_{4} u_{2} u_{3}+p_{291} u_{2} u_{3}^{2} \\
& +p_{216} u_{2} u_{3}-p_{190} u_{1} u_{3}+p_{290} u_{3}^{3} \\
& \left.-p_{292} u_{2} u_{1} u_{3}+p_{295} q_{4} u_{3}^{2}\right) .
\end{aligned}
$$

In eqn (11), $q_{4}$ is the articulation angle between tractor and semitrailer, $u_{1}, u_{2}$, and $u_{3}$ are generalized speeds, and the various $p$ symbols $\left(p_{239}, p_{173}\right.$, etc. $)$ are symbols introduced to replace expressions involving only constants.

When building these equations, it is necessary to keep track of many terms that do not appear in the final form. The 'bookkeeping' of intermediate terms places high demands on computer memory. Further, the many comparisons and sorting operations needed to manipulate and combine complex expressions require significant computer time. The overhead associ- ated with intermediate terms has long been a practical problem associated with symbolic computation that is often called 'equation swell'. To avoid equation swell, it is recommended that expressions be simplified as soon as possible, both by (1) dropping higher-order terms, and (2) combining remaining terms and replacing constant expressions with new symbols. Note that in eqn (11), all terms are of order three or less, and all coefficients not involving the state variables have been absorbed into coefficients that were introduced automatically.

The design of AUTOSIM is such that all symbolic expressions have about a dozen associated attributes in addition to the purely algebraic representation. These attributes are used to support the various symbolic algebra and calculus operations. Two attributes that are of particular interest here are: (1) identification as to whether the expression is a constant or a variable, and (2) a 'small-order' value. The 'constant or variable' information is used to factor out coefficients that are constants, to produce expressions such as the right-hand side of eqn (11).

Two 'rules' are programmed for dropping higherorder terms. In both cases, an expression is dropped when a comparison of its small-order value with some threshold indicates that it is negligible. The standard rule is that high-order terms are dropped when they are added to a term and the difference between the small-order values of the terms is greater than or equal to the threshold. A simpler rule, used for the analyses described in this paper, is that highorder terms are not even created if the small-order value would be greater than or equal to the threshold. (The threshold is set by the analyst. For example, if third order terms are to be kept, the threshold is four.)

Given that the objective of the symbolic analysis is eqn (2), with the right-hand side expanded in a power series in terms of the local state variables, the general strategy is to perform power series expansions at the first opportunity. For trigonometric functions, the power series are applied to the argument if it has a small-order value greater than zero. (It turns out that for the vehicle systems of interest, the arguments to trigonometric functions are always expressions whose small-order value is at least one.) For the division operation, the expansion used is

$$
\frac{1}{1-x}=1+x+x^{2}+x^{3}+\ldots
$$

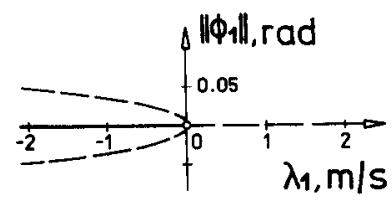

Fig. 4. Amplitude branch of an unstable limit cycle $\left(\lambda_{1}=V-V_{c}, d_{1}=1.104 \mathrm{~m},-\right.$ stable, - - unstable). 
$\phi_{1}, \mathrm{rad}$

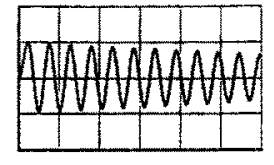

Time, s

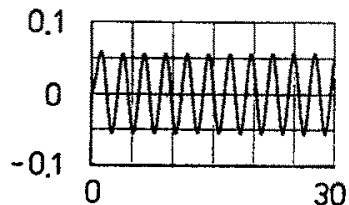

b

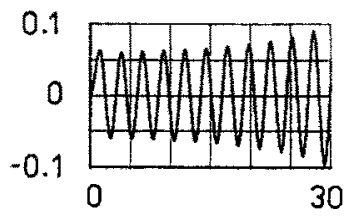

C

Fig. 5. Simulation results of transient vehicle motions at the same constant forward speed $\left(\lambda_{1}=-2 \mathrm{~m} / \mathrm{s}\right)$.

where $|x|<1$. In the general case of $1 / y$, where $y$ is a sum of terms, it is necessary to separate $y$ into two parts: a part with the same small-order value as $y$, and a part with higher order (i.e. smaller) terms. For example, consider $y=a+b$, where $a$ represents the sum of terms having the same small-order value as $y$, and $b$ represents the sum of terms whose small-order values are higher. The expression $1 / y$ is expanded as

$$
\begin{aligned}
\frac{1}{y}=\frac{1}{a+b}=\frac{1}{a}\left(\frac{1}{1+b / a}\right) & \\
& =\frac{1}{a}-\frac{b}{a^{2}}+\frac{b^{2}}{a^{3}}-\frac{b^{3}}{a^{4}}+\ldots
\end{aligned}
$$

One way to take a sum to an integer power is to apply all multiplications and then drop terms whose small-order values are equal or above the threshold. This approach is not recommended, because it accentuates 'equation swell'. For example, in an analysis with fifth order terms, a polynomial taken to the fifth power will have terms up to order 25 . Because all terms from order 6 to 25 will eventually be thrown out, any effort spent manipulating them is wasted.

A better method for raising a sum to an integer power is to drop terms as each multiplication is performed. An even more efficient method, which is the one used in AUTOSIM, is to use a solution that has already been worked out for each integer power. For example, consider an expression of the form: $x=(a+b)^{2}$. Rather than going through the intermediate step ' $x=a^{2}+a b+a b+b^{2}$ and then reducing it to ' $x=a^{2}+2 a b+b^{2}$, the expressions represented by $a$ and $b$ can be substituted directly into the solution. For powers higher than two, and a large number of terms in the sum, considerable savings in time and computer memory are achieved. However,

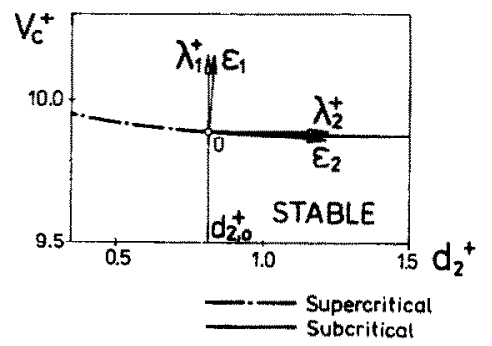

Fig. 6. Stability boundary in the parameter space $\left(V^{+}=V / \sqrt{ }(g s), d_{2}^{+}=d_{2} / s, d_{1}=1.104 \mathrm{~m}\right)$. it is necessary to have worked out and programmed the form of the solution beforehand. In AUTOSIM, solutions have been programmed for powers up to five. (Higher powers are decomposed into powers of five and less.)

The strategy of expanding expressions as they are encountered is not always best. Sometimes, significant simplifications can be made if nonlinear expressions are kept in their original form. This is generally the case when deriving expressions for angles, because the derivation requires normalizing vectors. For example, the normalized velocity vector for the left-front wheel location (point $P 1$ ), is

$$
\hat{\nabla}=\frac{\mathbf{v}^{p_{1}}}{\left|\mathbf{v}^{p \mid}\right|}=\frac{\left(V+p_{1} u_{2}\right) \mathbf{a}_{1}+\left(u_{1}+c_{0} u_{2}\right) \mathbf{a}_{2}}{\sqrt{\left[\left(V+p_{1} u_{2}\right)^{2}+\left(u_{1}+c_{0} u_{2}\right)^{2}\right]}}
$$

where $a_{1}$ and $a_{2}$ are unit-vectors fixed in body $A$ (the tractor), and $v^{P_{1}}$ is the velocity of the point $P 1$. This term is used to derive a slip angle by taking the ratio of the dot-product of and $a_{2}$ and $p$ and $a_{1}$

$$
\begin{aligned}
\alpha_{1} & =\tan ^{-1}\left(\boldsymbol{\theta} \cdot \mathbf{a}_{2} / \boldsymbol{v} \cdot \mathbf{a}_{1}\right) \\
& =\tan ^{-1}\left(c_{0} u_{2}+u_{1}\right) /\left(V+p_{1} u_{2}\right) .
\end{aligned}
$$

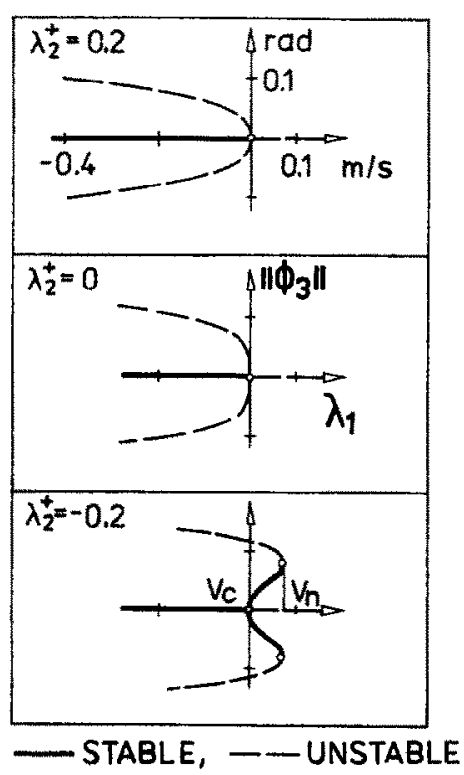

Fig. 7. Global limit cycle branches at different loading conditions of the second trailer $\left(\lambda_{1}=V-V_{c}, \lambda_{2}^{+}=\right.$ $d_{2}^{+}-d_{2,0}^{+}$; - stable, - unstable). 
In the tire model of eqn (1), only the tangent of $\alpha$ appears. Thus, the expression in eqn (15) is simplified because it is defined in terms of the inverse tangent function.

The point being made here is that when expressions are formed for the tangents of the slip angles, the power-series expansions are not used at each step of the way. The full, nonlinear expressions are derived to exploit potential symbolic simplifications. Once the tangent function is obtained, the expression is expanded into a power series. For example, eqn (15) leads to the expansion

$$
\begin{aligned}
\tan \alpha_{1}= & c_{0} u_{2} / V-c_{0} p_{1} u_{2}^{2} / V^{2}+c_{0} p_{1}^{2} u_{2}^{3} / V^{3} \\
& +p_{1}^{2} u_{1} u_{2}^{2} / V^{3}-p_{1} u_{2} u_{1} / V^{2}+u_{1} / V
\end{aligned}
$$

\section{NUMERICAL RESULTS}

\subsection{Tractor-semitrailer}

Fortran source code is generated automatically to compute all of the coefficients implied by eqn (2) up to the third order. The stability analysis is then performed numerically by a program that includes that Fortran code. Depending on the loading condition, the stability boundary for a tractorsemitrailer is shown in Fig. 3. At the critical speed, $V_{c}$, we always found an unstable limit cycle bifurcating from the steady-state straightline motion. For a certain loading condition the amplitude of the unstable limit cycle calculated from eqn (7) with $\dot{r}=0$ is shown in Fig. 4. The limit cycle bounds the domain of attraction of the stable straightline motion. Figure 5 shows results from computer simulations following the bifurcation analysis. For the simulations we have used the full, nonlinear simulation code generated by AUTOSIM. The results are in agreement with the results obtained from the bifurcation analysis. Due to a subcritical Hopf bifurcation, a nonlinear unstable vehicle behavior can occur at speeds lower than the critical speed, caused by a disturbance. (See Fig. 5, case c.)

\subsection{Global stability investigation of a twin-trailer truck}

Using AUTOSIM, the coefficients implied by eqn (2) were derived for the twin-trailer vehicle shown in Fig. 1. To perform a global stability analysis, terms up to the fifth order were kept. The equations for this system cover over 150 pages when written out. Thus, computer algebra was essential in the global analysis of the straightline stability of this vehicle system.

For the loading conditions considered, we found that the loading condition of the second trailer does not have a strong influence on that stability boundary, which also was found for the vehicle combination without second trailer, (see Fig. 3). Figure 6 shows the weak dependence of the critical speed on the distance from the mass center of the second semitrailer to its axle, $d_{2}$ (see Fig. 1). However, smaller values of $d_{2}$ can indeed lead to a completely different situation [1].

For the tractor-semitrailer, only a subcritical bifurcation behavior has been found. However, for the twin-trailer vehicle, the bifurcation behavior can change continuously, from subcritical to supercriti$\mathrm{cal}$, as a function of $d_{2}$. Accordingly, we can adjust this parameter to the nongeneric point, where the subcritical Hopf bifurcation becomes supercritical. In Fig. 6 the directions $\left(\epsilon_{1}, \epsilon_{2}\right)$ in the parameter space for the unfolding of the nongeneric case are shown. From the unfolded eqn (9) with $\dot{r}=0$ we are now able to calculate the global amplitude branches of the limit cycles at different loading conditions of the second trailer.

Figure 7 shows the calculated amplitudes of the articulation angle, $\phi_{3}$, between the center lines of the lead unit and the second semitrailer. We see that, at certain loading conditions, a stable limit cycle motion of the twin-trailer truck is possible beyond the critical speed, $V_{c}$, up to a nonlinear speed, $V_{n}$. The unstable limit cycle branch bounds the attraction domain of the stable steady-state straightline motion, or of the stable periodical motion, respectively.

Acknowledgement-This work has been supported in part by the U.S. Army Tank Automotive Command, and in part by the Austrian Science Foundation (FWF-Project No. J0344-TEC).

\section{REFERENCES}

1. A. Stribersky and P. S. Fancher, The nonlinear behavior of heavy-duty truck combinations with respect to straightline stability. ASME J. Dyn. Syst., Measure ments, Control 111, 577-582 (1989).

2. D. Moelle and R. Gasch, Nonlinear bogie hunting. Proc. 7th IAVSD Symposium, Cambridge, U.K., pp. $455-467$ (Edited by A. H. Wickens), Swets \& Zeitlinger, Lisse (1982).

3. V. I. Arnold, Geometrical Methods in the Theory of Ordinary Different Equations. Springer, Heidelberg (1983).

4. J. Carr, Applications of centre manifold theory. Applied Math. Sciences 35. Springer, New York (1981).

5. J. Guckenheimer and P. Holmes, Nonlinear oscillations, dynamical systems, and bifurcation of vector fields. Applied Math. Sciences 42. Springer, New York (1983).

6. M. W. Sayers, Symbolic computer methods to automatically formulate vehicle simulation codes. Ph.D. thesis, University of Michigan (1990).

7. V. Kaçani, A. Stribersky, H. Troger and K. Zeman, Dynamics and bifurcations in the motion of tractorsemitrailer vehicles. Conference Proceed. Canadian Math. Soc., Vol. 8, (Edited by F. V. Atkinson, W. F. Langford and A. B. Mingarelli), pp. 485-499 (1987).

8. A. Stribersky, P. S. Fancher, C. C. MacAdam and M. W. Sayers, On nonlinear oscillations in road trains at high forward speeds. Proc. 1lth IAVSD Symp., Kingston, Ontario, (Edited by R. Anderson). Swets \& Zeitlinger, Amsterdam (1989).

9. A. Schallamach and D. M. Turner, The wear of slipping wheels. Wear 3, 1-25 (1960).

10. J. Wittenburg, Dynamics of Systems of Rigid Bodies. B. G. Teubner, Stuttgart (1977). 
11. W. O. Schiehlen, Dynamics of complex multibody systems. Solid Mech. Arch. 9, 159-195 (1984).

12. R. E. Roberson and R. Schwertassek, Dynamics of Multibody Systems. Springer, Berlin (1988).

13. N. Orlandea, M. A. Chace and D. A. Calahan, A sparsity-oriented approach to the dynamic analysis and design of mechanical systems, parts I and II. $J$. Engng Ind. 99, 773-784 (1977).

14. P. E. Nikravesh and E. J. Haug, Generalized coordinate partitioning for analysis of mechanical systems with nonholonomic constraints. ASME J. Mech., Trans. Automat. Design 105, 379-384 (1983).

15. D. E. Rosenthal and M. A. Sherman, High performance multibody simulations via symbolic equation manipu- lation and Kane's method. J. Astron. Sci. 34, 223-239 (1986).

16. G. I. J. Steele, Common Lisp: The Language. Digital Press (1984).

17. T. R. Kane and D. A. Levinson, Dynamics, Theory and Applications, McGraw-Hill Series in Mechanical Engineering. McGraw-Hill (1985).

18. S. N. Chow and J. Mallet-Paret, Integral averaging and bifurcation. J. Differential Eqn 6, 112-159 (1977).

19. A. Stribersky, Nichtlineare Stabilitätsuntersuchungen dynamischer Systeme, (Nonlinear stability investigations of dynamical systems). Fortschritt-Berichte VDI, Reihe 11, Nr. 97. VDI Verlag, Düsseldorf (1987).

\section{APPENDIX}

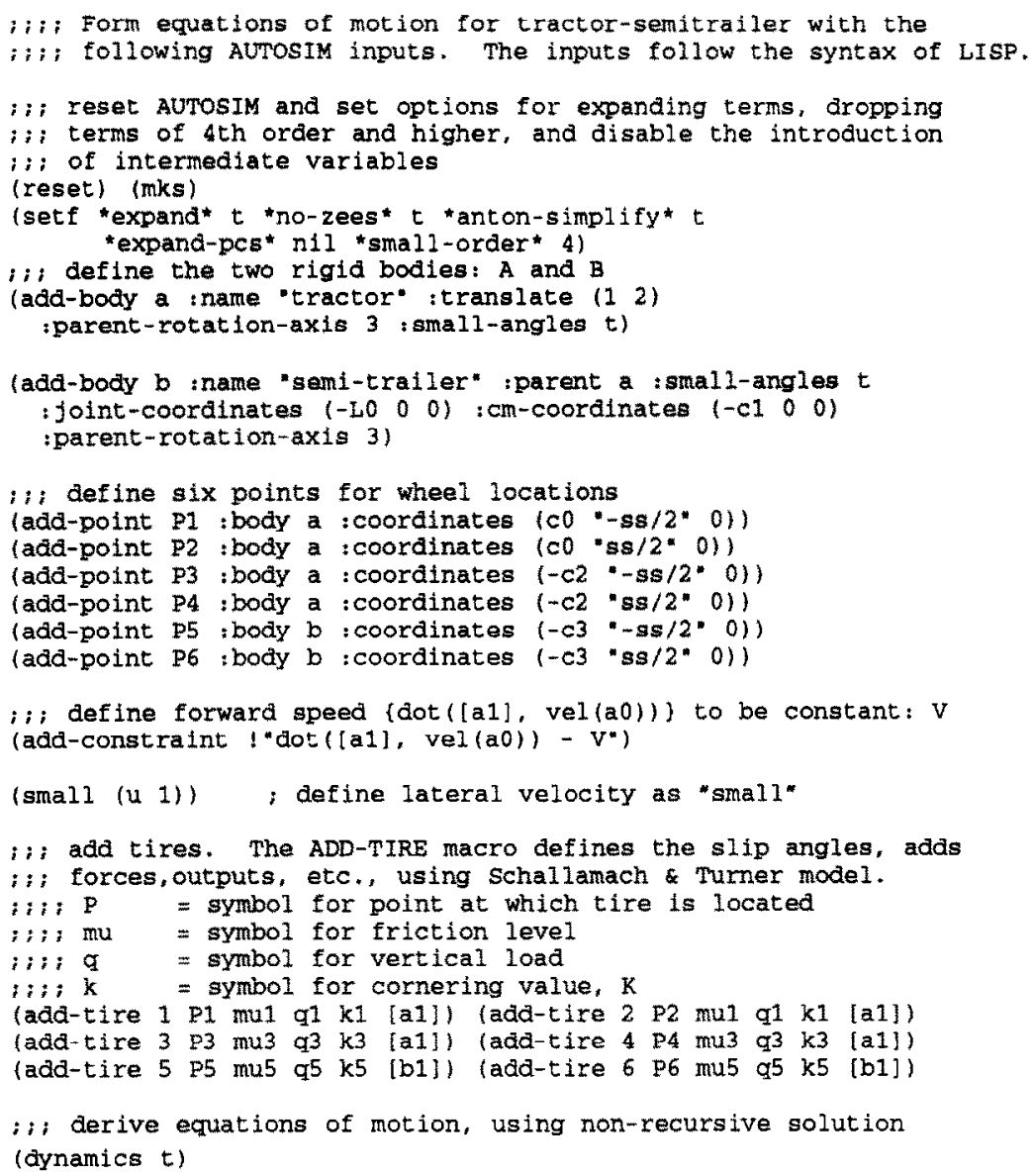

